\title{
Is bronchoscopy necessary in the preoperative workup of a solitary pulmonary nodule?
}

\author{
Yiliang Zhang, MD, ${ }^{\mathrm{a}, \mathrm{b}}$ Yang Zhang, MD, ${ }^{\mathrm{a}, \mathrm{b}}$ Sufeng Chen, MD, ${ }^{\mathrm{a}, \mathrm{b}}$ Yuan Li, PhD, ${ }^{\mathrm{b}, \mathrm{c}}$ Yongfu Yu, MD, \\ Yihua Sun, $\mathrm{PhD},{ }^{\mathrm{a}, \mathrm{b}}$ and Haiquan Chen, $\mathrm{MD}^{\mathrm{a}, \mathrm{b}}$
}

\begin{abstract}
Objective: This study evaluated the role of conventional bronchoscopy in the preoperative workup of patients with solitary pulmonary nodules (SPNs).

Methods: Patients with SPNs of unknown origin were enrolled for preoperative bronchoscopy at our institution. Bronchoscopic findings were prospectively collected, and their impact on planned therapy was analyzed.
\end{abstract}

Results: A total of 1026 patients were included. Bronchoscopy identified unsuspected findings in $80(7.8 \%)$ of them, with a total of $826(80.5 \%)$ malignant nodules. Referent values for bronchoscopic detection of malignant SPNs were: accuracy, $24.3 \%$ (95\% confidence interval [CI]: 21.7\%-27.0\%); sensitivity, $5.9 \%$ (95\% CI: $4.5 \%-7.4 \%$ ); specificity, $100 \%$; and negative predictive value, $20.5 \%$ (95\% CI: $18.0 \%-22.9 \%)$. Malignant bronchoscopic findings were more common in male patients (odds ratio $[\mathrm{OR}]=2.1,95 \% \mathrm{CI}: 1.1-3.9, P=.021$ ) and large nodules $(\mathrm{OR}=2.3,95 \% \mathrm{CI}$ : $1.6-3.3, P<.001)$. Surgery was cancelled in $2(0.2 \%)$ patients and modified in $36(3.5 \%)$ patients because of bronchoscopic findings. In all, for $268(26.1 \%)$ SPNs that presented with ground-glass opacity, the bronchoscopy was unrevealing.

Conclusions: In the preoperative evaluation of SPN, bronchoscopy is most likely to reveal malignancy in larger SPNs and in male patients. Bronchoscopy is not indicated in SPNs that present with ground-glass opacity on computed tomography scan. (J Thorac Cardiovasc Surg 2015;150:36-40)

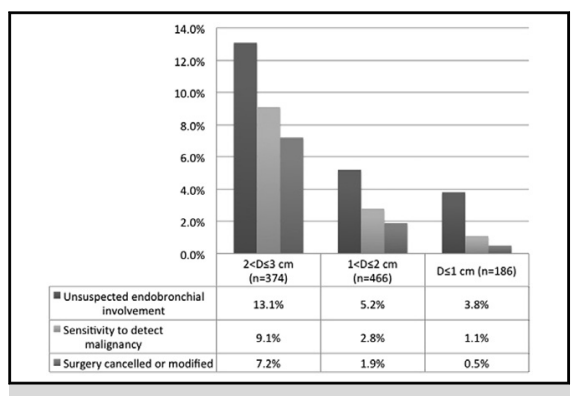

Yields from bronchoscopy decreased significantly with nodule size.

\section{Central Message}

Bronchoscopy identified unsuspected findings in $7.8 \%$ of patients with solitary pulmonary nodules, altering therapy in $3.7 \%$. Preoperative bronchoscopy provides greatest benefit with men, and larger nodules.

\section{Perspective}

Appropriateness of routine bronchoscopy in preoperative workup of solitary pulmonary nodules (SPNs) is debatable. Our findings show that preoperative bronchoscopy reveals malignancy most often with men, and larger SPNs; its utility is limited with women, and SPNs presenting with ground-glass opacity on computed tomography scan. The data argue against routine bronchoscopy for women with small SPNs, unless central lesions are highly suspected.

See Editorial Commentary page 40.
The appropriateness of routine use of bronchoscopy in the preoperative workup of solitary pulmonary nodule (SPNs) is debatable. Opinions expressed in the literature vary, ranging from the idea that routine preoperative bronchoscopy has no role in conducting tissue diagnosis in small SPNs, ${ }^{1}$ to the idea that it is very useful in determining underlying etiology and surgical strategy. ${ }^{2}$ The American College of Chest Physicians guidelines ${ }^{3}$ recommend bronchoscopy only if an

From the Department of Thoracic Surgery, ${ }^{a}$ Fudan University Shanghai Cancer Center, Shanghai; Department of Oncology, ${ }^{\mathrm{b}}$ Shanghai Medical College, Fudan University, Shanghai; Department of Pathology, ${ }^{\mathrm{c}}$ Fudan University Shanghai Cancer Center, Shanghai, People's Republic of China; and Section of Epidemiology, Department of Public Health, Aarhus University, Aarhus, Denmark.

Received for publication Sept 6, 2014; revisions received Jan 25, 2015; accepted for publication Feb 3, 2015; available ahead of print April 2, 2015. air bronchogram is present or if the operator has expertise with newer, guided techniques. We hypothesize that bronchoscopy has a limited but useful role in the preoperative assessment of SPNs. The purpose of the current analysis is to define that role by examining, in our patients with SPNs: (1) endobronchial findings; (2) the impact of these findings on therapy; (3) referent values for malignant SPNs; and (4) variables that are associated with bronchoscopic findings.

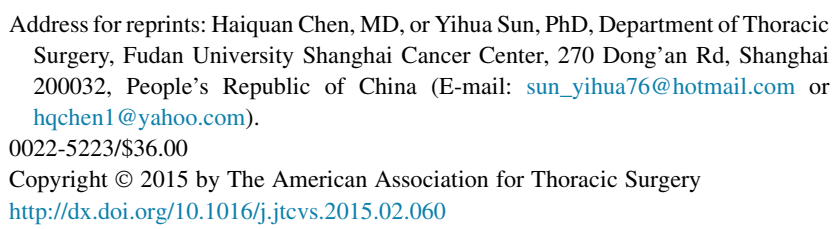




\section{Abbreviations and Acronyms \\ $\mathrm{CI}=$ confidence interval \\ $\mathrm{CT}=$ computed tomography \\ $\mathrm{OR}=$ odds ratio \\ $\mathrm{SPN}=$ solitary pulmonary nodule}

\section{METHODS}

\section{Patients}

This study was conducted in a prospective manner at the Department of Thoracic Surgery, Fudan University Shanghai Cancer Center, from January 2008 to December 2013. We defined an SPN as a single, wellcircumscribed radiographic opacity that measures $\leq 3 \mathrm{~cm}$ in diameter and is surrounded completely by aerated lung, with an absence of atelectasis, hilar enlargement, or pleural effusion. ${ }^{3}$ Exclusion criteria for patients were: (1) having a benign SPN, as determined by fine-needle biopsy, and $\geq 1$ year of follow up via thoracic computed tomography (CT); (2) refusing or not being suitable for surgery; (3) refusing bronchoscopy or being physically unable to undergo examination to evaluate the whole tracheobronchial tree (Figure 1).

Scan data from chest CT (64-row; slice width: $1.0 \mathrm{~mm}$ ) were acquired from all patients before bronchoscopy. Individual CT datasets were transferred to a workstation (Advantage Workstation 4.3, GE Healthcare, Barrington, Ill), on which 3-dimensional images of the SPN and adjacent bronchus were created. Bronchoscopic examination and biopsy were performed by the same thoracic surgeon. Transtracheal airway anesthesia was conducted using $2 \%$ lidocaine. Additional $2 \%$ lidocaine spray was instilled in the nares and throat to provide additional patient comfort.

A flexible bronchoscope was placed in the nose or mouth with the patient in a supine position. Once the bronchoscope was inserted into the upper airway, the vocal cords were inspected. The instrument was advanced to the larynx and further down into the tracheobronchial tree, and each area was inspected as the bronchoscope passed, including the subsegmental bronchi. The bronchoscopist selected the bronchial path to the nodule, based on the CT findings, when it seemed possible to reach the lesion. Biopsies were taken using a brush, needle, forceps, or cytologic washing.

Patients were later scheduled for surgery if the bronchoscopy produced negative results. Depending on the clinical and functional status of the patient, as well as the histologic and anatomic findings, either sublobar resection (wedge/segmental), lobectomy (including sleeve lobectomy), bilobectomy, or pneumonectomy was performed, after exclusion of distal metastases.

After screening, 1026 remaining patients were included in this study; characteristics and radiologic findings are presented in Table 1. Clinicopathologic data on age, gender, smoking history, nodule size measured on CT, type of surgery, and pathology were collected. This study was conducted in accordance with the Helsinki Declaration and approved by the institutional review board of the Fudan University Shanghai Cancer Center, Shanghai, People's Republic of China. All patients provided written informed consent.

\section{Statistical Analysis}

Statistical analysis was performed using SPSS (version 19.0, SPSS, Inc, Chicago, Ill). Continuous variables were expressed as the mean $\pm \mathrm{SD}$; categoric variables were expressed as frequency and percentage, for description. Comparison of continuous variables was examined using an independent Student $t$ test and the Mann-Whitney $U$ test.

Statistical analysis of categoric variables was performed using Pearson $\chi^{2}$ analysis and the Fisher exact test, as appropriate. Multivariate analysis was conducted using logistic regression to identify independent predictors. The endpoint is the bronchoscopic yield. The variables analyzed included gender, age, smoking status, and tumor size and location. All statistical analyses were 2 -sided.

\section{RESULTS \\ Bronchoscopic Results}

Positive bronchoscopic findings were established in 80 (7.8\%) patients, including 38 (3.7\%) with incidental endobronchial lesions, 4 with mucosa edema, 1 with variant segment, and $37(3.6 \%)$ in whom the SPN was reachable and biopsies were successfully taken. Histologically, in 37 SPNs successfully biopsied under bronchoscopy, 11 were determined to be malignant, and 26 were benign. For these 26 nodules, 1 was discovered, after surgery, to be adenocarcinoma (false negative).

In 38 cases of endobronchial lesions, bronchoscopic pathology revealed 25 cases of squamous cell cancer, 6 of adenocarcinoma, 3 of carcinoid, 2 of mucoepidermoid carcinoma, and 2 of small cell lung cancer. A total of 826 $(80.5 \%)$ malignant SPNs were diagnosed according to the pathologic reports of resected specimens, as well as bronchoscopic results. Therefore, referent values for bronchoscopic detection of malignant SPNs were: accuracy, $24.3 \%$ (95\% confidence interval [CI]: 21.7\%-27.0\%); sensitivity, $5.9 \% \quad(95 \%$ CI: $4.5 \%-7.4 \%)$; specificity, $100 \%$; positive predictive value, $100 \%$; and negative predictive value, $20.5 \%$ (95\% CI: $18.0 \%-22.9 \%$ ); Table 2 .

\section{Treatment}

Of the 1024 patients undergoing surgeries, video-assisted thoracoscopic surgery was conducted in $492(48.0 \%)$, and thoracotomy in $532(52.0 \%)$. The surgical approach had to be modified to: a sleeve lobectomy in 10 patients; a bilobectomy in 12 patients; pneumonectomy in 14 patients; and no surgery in 2 cases with small cell lung cancer, owing to the bronchoscopic findings. Therefore, surgeries had to be modified or cancelled in a total of $38(3.7 \%)$ patients (Table 3).

\section{Bronchoscopic Yield, by Parameter}

Univariate analysis revealed that bronchoscopic yield is related to gender, smoking status, and nodule size, but may not be associated with patient age and nodule location (Table 4). On multivariate analysis, the rate of positive bronchoscopic findings was significantly higher in men (odds ratio $[\mathrm{OR}]=2.277,95 \%$ CI: $1.380-3.757$, $P=.001)$ and large nodules $(\mathrm{OR}=2.425,95 \% \mathrm{CI}$ : 1.713-3.433, $P<.001$ ) (Table 4). Positive bronchoscopic findings were detected in $13.1 \%(\mathrm{n}=49)$ of nodules of $>2 \mathrm{~cm}$ and $\leq 3 \mathrm{~cm}$ in size. The prevalence decreased to $5.2 \%(\mathrm{n}=24)$ in the SPNs sized between $>1 \mathrm{~cm}$ and $\leq 2$ $\mathrm{cm}$, and $3.8 \%(\mathrm{n}=7)$ in the subcentimeter nodules.

The sensitivity of bronchoscopy to detect malignancy declined from $9.1 \%(\mathrm{n}=34)$, in lesions 2 to $3 \mathrm{~cm}$ in size, to $2.8 \%(\mathrm{n}=13)$ in nodules 1 to $2 \mathrm{~cm}$ in size, and to 


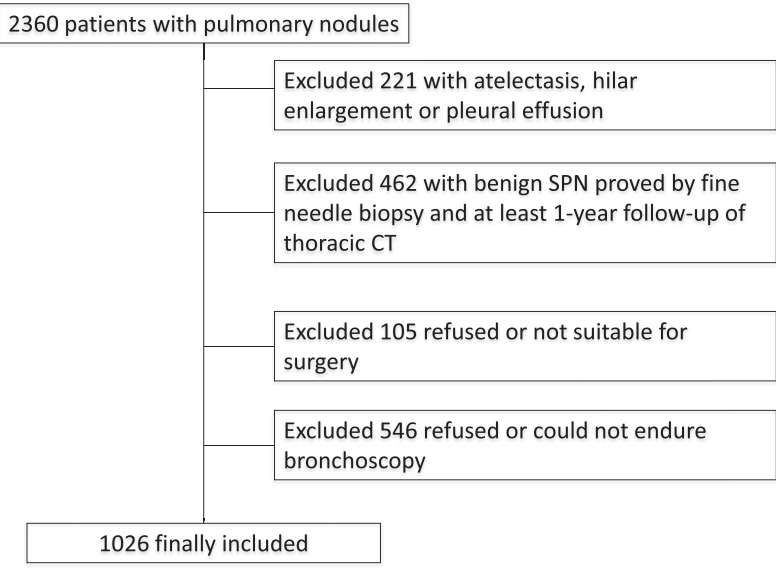

FIGURE 1. Criteria for selection and exclusion of subjects. $S P N$, Solitary pulmonary nodule; $C T$, computed tomography.

$1.1 \%(\mathrm{n}=2)$ in the subcentimeter group. Both of the 2 small cell lung tumors identified by bronchoscopic biopsy were $>2 \mathrm{~cm}$ in size. Of patients with SPNs between 2 and $3 \mathrm{~cm}$ in size, surgeries were cancelled or modified in 27 $(7.7 \%)$. The types of surgical resection were modified to sleeve lobectomy, bilobectomy, or pneumonectomy in 9 $(1.9 \%)$ patients who had tumors in the 1 to $2 \mathrm{~cm}$ category, and $1(0.5 \%)$ patient in the subcentimeter group (Figure 2). For the $268(26.1 \%)$ SPNs with a ground-glass-opacity component, the results of all the bronchoscopic workups were negative.

\section{DISCUSSION}

The incidence of SPNs is increasing, especially with increasing use of chest CT scanning for diagnosis of a multitude of chest diseases. The National Lung Screening Trial documented that screening for lung cancer in current and

TABLE 1. Patient characteristics

\begin{tabular}{lc}
\hline \multicolumn{1}{c}{ Characteristic } & Patients $(\mathbf{n}=\mathbf{1 0 2 6})$ \\
\hline Gender (male/female) & $550 / 476$ \\
Age (mean \pm SD, y) & $58.4 \pm 10.5$ \\
Smoking history & 425 \\
Nodules contain GGO component & 268 \\
Nodule location & \\
$\quad$ Left, upper lobe & 261 \\
Left, lower lobe & 157 \\
Right, upper lobe & 343 \\
Right, middle lobe & 80 \\
Right, lower lobe & 185 \\
Size of nodules on CT (mean $\pm \mathrm{SD}$, cm) & $1.88 \pm 0.73$ \\
Nodule size (cm) & \\
$\leq 1$ & 186 \\
$>1, \leq 2$ & 466 \\
$>2, \leq 3$ & 374 \\
\hline$G G O$, Ground-glass opacity; $C T$, computed tomography; $S D$, standard deviation.
\end{tabular}

TABLE 2. Sensitivity of bronchoscopy to detect SPN malignancy

\begin{tabular}{lccc}
\hline \multicolumn{1}{c}{ Histologic type } & $\begin{array}{c}\text { Bronchoscopic } \\
\text { pathology } \\
(\mathbf{n = 4 9 )}\end{array}$ & $\begin{array}{c}\text { Final } \\
\text { pathology } \\
(\mathbf{n = 8 2 6})\end{array}$ & $\begin{array}{c}\text { Sensitivity } \\
(\%)\end{array}$ \\
\hline Lung cancer & 48 & 753 & 6.4 \\
Adenocarcinoma & 9 & 561 & 1.6 \\
Squamous cell carcinoma & 32 & 108 & 29.6 \\
AIS & 0 & 24 & 0 \\
Small cell carcinoma & 2 & 13 & 15.3 \\
ASC & 0 & 12 & 0 \\
AAH & 0 & 11 & 0 \\
Large cell carcinoma & 0 & 8 & 0 \\
Carcinoid & 3 & 8 & 37.5 \\
Mucoepidermoid carcinoma & 2 & 7 & 28.5 \\
Sarcomatoid carcinoma & 0 & 1 & 0 \\
Lymphoma & 0 & 2 & 0 \\
Metastases & 1 & 71 & 1.4 \\
\hline AIS, Adenocarcinoma in situ; $A S C$, adenosquamous carcinoma; $A A H$, atypical adeno- \\
matous hyperplasia.
\end{tabular}

former smokers using a low-dose chest $\mathrm{CT}$ scan could reduce lung cancer-specific mortality by $20 \%$, and that surgical resection is a predominant method for the treatment of malignant SPNs and reduction of associated mortality. ${ }^{4,5}$ Controversy remains over the role of conventional bronchoscopy in the evaluation of the SPN. Pulmonologists and thoracic surgeons may have differing opinions on this issue. Pulmonologists are inclined to demand a tissue diagnosis, and to clarify the underlying etiology via bronchoscopy; surgeons need the examination as a means to exclude operative contraindications and make treatment plans.

In this study, bronchoscopy was used as the routine preoperative evaluation of SPNs suspected of being malignant. The yields of bronchoscopy for SPNs have been investigated mainly in terms of positive findings, sensitivity to detect malignancy, and alteration of surgical strategies. Results show that preoperative bronchoscopy is most likely to reveal malignancy in male patients and those with large nodules, but bronchoscopy utility is limited for female patients who have SPNs, or those who have ground-glass opacity on CT scan. This difference may be explained by the fact that central bronchial involvement is more common in men than in women. ${ }^{6}$ The data make a reasonable argument against

TABLE 3. Treatment procedures and those being modified according to the results of bronchoscopy

\begin{tabular}{lcc}
\hline \multicolumn{1}{c}{ Treatment } & $\begin{array}{c}\text { All patients } \\
(\mathbf{n = 1 0 2 6})\end{array}$ & $\begin{array}{c}\text { Patients with treatment } \\
\text { being modified }(\mathbf{n}=\mathbf{3 8})\end{array}$ \\
\hline Sublobar resection & 311 & 0 \\
Lobectomy & 655 & 0 \\
Sleeve lobectomy & 12 & 10 \\
Bilobectomy & 32 & 12 \\
Pneumonectomy & 14 & 14 \\
Surgery cancelled & 2 & 2 \\
\hline
\end{tabular}


TABLE 4. Predictors of positive bronchoscopic findings

\begin{tabular}{lcccc}
\hline \multicolumn{1}{c}{ Type of analysis } & $\begin{array}{c}\text { Positive } \\
\text { no. (\%) }\end{array}$ & $\begin{array}{c}\text { Odds } \\
\text { ratio }\end{array}$ & $\mathbf{9 5 \% \text { CI }}$ & $\begin{array}{c}\boldsymbol{P} \\
\text { value }\end{array}$ \\
\hline Univariate & & & & \\
$\quad$ Gender, male/female & $57(10.4) / 23(4.8)$ & 2.277 & $1.380-3.757$ & .001 \\
$\quad$ Age & - & 0.998 & $0.977-1.020$ & .877 \\
$\quad$ Smoker, never vs ever & $38(6.3) / 42(9.9)$ & 0.615 & $0.389-0.973$ & .038 \\
Nodule size & - & 2.425 & $1.713-3.433$ & .000 \\
Nodule location & & & & \\
$\quad$ Left, upper lobe & $20(7.7)$ & - & - & .904 \\
$\quad$ Left, lower lobe & $12(7.6)$ & 0.877 & $0.441-1.741$ & .707 \\
$\quad$ Right, upper lobe & $24(7)$ & 0.874 & $0.400-1.908$ & .736 \\
$\quad$ Right, middle lobe & $8(10)$ & 0.795 & $0.411-1.537$ & .495 \\
$\quad$ Right, lower lobe & $16(8.6)$ & 1.174 & $0.481-2.865$ & .725 \\
Multivariate & & & & \\
$\quad$ Gender, male/female & $57(10.4) / 23(4.8)$ & 2.070 & $1.114-3.848$ & .021 \\
$\quad$ Smoker, never vs ever & $38(6.3) / 42(9.9)$ & 1.021 & $0.578-1.804$ & .943 \\
$\quad$ Nodule size & & 2.325 & $1.639-3.300$ & .000 \\
\hline$C I$, Confidence interval. & & & &
\end{tabular}

routine bronchoscopy for women who have a small SPN, unless central lesions are highly suspected.

Goldberg and colleagues ${ }^{7}$ retrospectively reviewed the utility of bronchoscopy in 33 SPNs, and revealed no unsuspected endobronchial lesion. Torrington and $\mathrm{Kern}^{8}$ found additional submucosal or endobronchial tumor manifestations in 5 of $91(5.5 \%)$ SPNs. However, those findings did not alter surgical strategies. Chhajed and colleagues ${ }^{9}$ combined bronchoscopy and positron emission tomography for the diagnosis of 74 patients with a small pulmonary nodule $(\leq 3 \mathrm{~cm})$. Endobronchial lesions were observed in 6 patients $(8.1 \%)$, but the effect on surgical strategy alteration was not mentioned. In a recent study, Schwarz and colleagues $^{2}$ prospectively performed preoperative flexible bronchoscopy in 225 patients who had SPNs, and they detected unsuspected endobronchial involvement in $4.4 \%$. Surgery was cancelled or modified in 2 cases, owing to those findings.

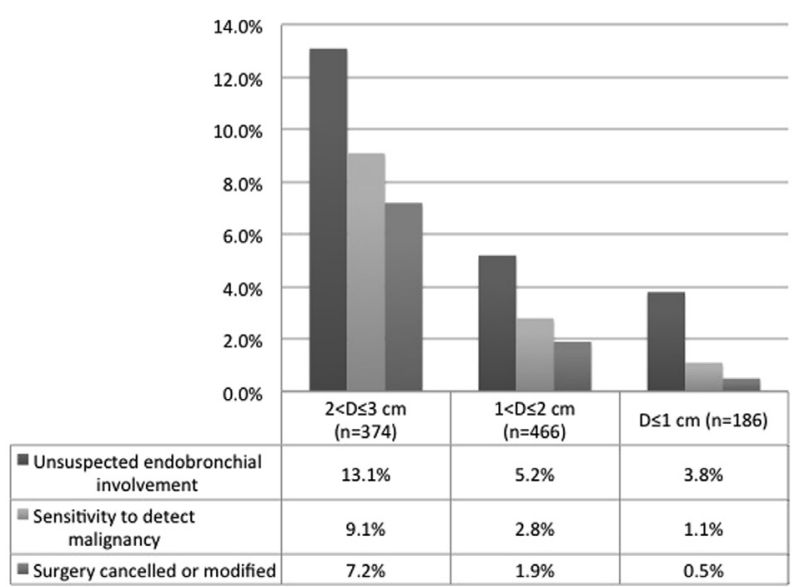

FIGURE 2. Yields from bronchoscopy decreased significantly with nodule size. $D$, Diameter.
The value of conventional bronchoscopy to obtain biopsies for pathologic analysis varied according to study population and combination strategy. In a study by van 't Westeinde and colleagues, ${ }^{10}$ investigation was made of the role of conventional bronchoscopy in the workup of 308 patients who had suspicious CT scan screen-detected pulmonary nodules; the sensitivity of bronchoscopy to detect malignancy was $13.5 \%$. In the prospective study conducted by Schwarz and colleagues, ${ }^{2}$ the sensitivity of flexible bronchoscopy to detect lung cancer was reported to be $46.5 \%$. One patient with small cell lung cancer who was diagnosed via bronchoscopy was eventually excluded from surgical resection. ${ }^{2}$ When combined with positron emission tomography, bronchoscopy had a diagnostic yield of $>50 \%$, as demonstrated by Chhajed and colleagues. ${ }^{9}$ Although the diagnostic value of bronchoscopy was relatively low in the current study, 2 patients with small cell lung cancer diagnosed via bronchoscopy were excluded from surgical resection.

\section{Recommendations and Limitations}

Our study has several limitations. First, positron emission tomography and/or CT was applied in only a small proportion of patients, and was therefore not included in this study. The preoperative diagnostic yields of bronchoscopy might be changed with the addition of positron emission tomography scanning. Second, we used conventional bronchoscopy. No fluoroscopy or any other navigational tool was used to guide transbronchial biopsy, and this factor may have made bronchoscopy less effective in obtaining a biopsy.

\section{CONCLUSIONS}

We report on 1026 patients who underwent bronchoscopy for preoperative evaluation of an SPN. Results implied that bronchoscopy is most likely to be beneficial in men and those with large nodules, and that bronchoscopy utility is limited for women with SPNs, or those nodules that present with ground-glass opacity on CT scan.

\section{Conflict of Interest Statement}

Authors have nothing to disclose with regard to commercial support.

\section{References}

1. Silvestri GA. Bronchoscopy for the solitary pulmonary nodule: friend or foe? Chest. 2012;142:276-7.

2. Schwarz C, Schonfeld N, Bittner RC, Mairinger T, Rüssmann H, Bauer TT, et al. Value of flexible bronchoscopy in the pre-operative work-up of solitary pulmonary nodules. Eur Respir J. 2013;41:177-82.

3. Gould MK, Donington J, Lynch WR, Mazzone PJ, Midthun DE, Naidich DP, et al. Evaluation of individuals with pulmonary nodules: When is it lung cancer? Diagnosis and management of lung cancer, 3rd ed: American College of Chest Physicians evidence-based clinical practice guidelines. Chest. 2013;143(5 Suppl):e93S-120S.

4. International Early Lung Cancer Action Program I, Henschke CI, Yankelevitz DF, Libby DM, Pasmantier MW, Smith JP, Miettinen OS. Survival of patients with stage I lung cancer detected on CT screening. N Engl J Med. 2006;355:1763-71. 
5. National Lung Screening Trial Research Team, , Aberle DR, Adams AM, Berg CD Black WC, Clapp JD, Fagerstrom RM. Reduced lung-cancer mortality with lowdose computed tomographic screening. New Engl J Med. 2011;365:395-409.

6. World Health Organization, International Agency for Research on Cancer, International Association for the Study of Lung Cancer, International Academy of Pathology. In: Travis WD, ed. Pathology and Genetics of Tumours of the Lung, Pleura, Thymus and Heart. Lyon Oxford: IARC Press; Oxford University Press; 2004.

7. Goldberg SK, Walkenstein MD, Steinbach A, Aranson R. The role of staging bronchoscopy in the preoperative assessment of a solitary pulmonary nodule. Chest. 1993;104:94-7.

8. Torrington KG, Kern JD. The utility of fiberoptic bronchoscopy in the evaluation of the solitary pulmonary nodule. Chest. 1993;104:1021-4.
9. Chhajed PN, Bernasconi M, Gambazzi F, Bubendorf L, Rasch H, Kneifel S, et al. Combining bronchoscopy and positron emission tomography for the diagnosis of the small pulmonary nodule $<$ or $=3 \mathrm{~cm}$. Chest. 2005;128: 3558-64.

10. van't Westeinde SC, Horeweg N, Vernhout RM, Groen HJ, Lammers JW, Weenink C, et al. The role of conventional bronchoscopy in the workup of suspicious CT scan screen-detected pulmonary nodules. Chest. 2012;142: 377-84.

Key Words: solitary pulmonary nodule, bronchoscopy, preoperative workup

\title{
EDITORIAL COMMENTARY
}

\section{Conventional bronchoscopy for evaluation of the solitary pulmonary nodule: Beneficial for a select few patients, or are we still "looking for a needle in a haystack"?}

\author{
Kenneth A. Kesler, MD
}

See related article on pages $36-40$.

Not only can evaluation of the solitary pulmonary nodule (SPN) be vexing, but SPN evaluations are also increasing as a result of the expanding use of computed tomographic (CT) imaging. Clinicians typically take multiple factors into consideration to get a general impression of malignancy risk, including patient age, smoking history, CT characteristics, and positron emission tomographic imaging findings. Calculated risk models have even been established in an attempt to assign chances of SPN malignancy more precisely. ${ }^{1}$ Removal through a minimally invasive approach of any nodule larger than $8 \mathrm{~mm}$ highly suspected to contain a primary pulmonary malignancy, followed by anatomic pulmonary resection with mediastinal lymph node sampling if non-small cell lung cancer is confirmed

From the Thoracic Division, Department of Surgery, Indiana University, Indianapolis, Ind.

Disclosures: Author has nothing to disclose with regard to commercial support.

Received for publication Feb 12, 2015; accepted for publication Feb 12, 2015; available ahead of print March 24, 2015.

Address for reprints: Kenneth A. Kesler, MD, Thoracic Division, Department of Surgery, Indiana University, Barnhill Dr, EM \#212, Indianapolis, IN 46202

(E-mail: kkesler@iupui.edu).

J Thorac Cardiovasc Surg 2015;150:40-1

$0022-5223 / \$ 36.00$

Copyright (c) 2015 by The American Association for Thoracic Surgery

http://dx.doi.org/10.1016/j.jtcvs.2015.02.042 by frozen-section pathologic review, is considered an optimal strategy for most patients with low surgical risk. ${ }^{2}$ Removal obviously eliminates sampling error inherent in needle biopsy, avoids delay in surgical treatment for patients in whom non-small cell lung cancer is confirmed, and is usually undertaken at low risk levels. For patients

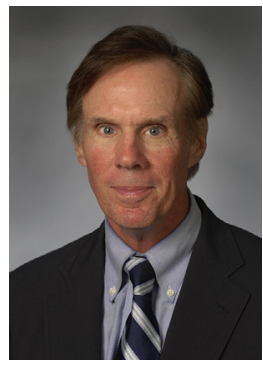
with SPNs suspected of malignancy who are reluctant to undergo any surgical intervention before a definitive diagnosis of malignancy, similar patients at higher surgical risk, or patients with SPNs at intermediate risk for malignancy, preoperative biopsy can be useful. Options in these cases include CT-guided needle biopsy or bronchoscopy with brushing, washing, or needle biopsy (unguided or guided) according to SPN location, SPN size, and institutional preference.

In this issue of the Journal, Zhang and coworkers ${ }^{3}$ report on 1026 patients who underwent conventional bronchoscopy for the evaluation of a SPN at their institution. Overall accuracy and negative predictive value in this study were $24.3 \%$ and $20.5 \%$, respectively, reinforcing the very wellestablished need for further investigation of suspect SPN when bronchoscopy results are negative. Higher diagnostic rates were found in male patients and with larger nodules. Conventional bronchoscopy resulted in a $7.8 \%$ overall incidence of positive diagnostic findings, including "altering therapy" in $3.7 \%$ of their series. 